\title{
Calibration of non-ideal thermal conductivity sensors
}

\author{
N. I. Kömle, W. Macher, G. Kargl, and M. S. Bentley \\ Space Research Institute, Austrian Academy of Sciences, Graz, Austria \\ Correspondence to: N. I. Kömle (norbert.koemle@oeaw.ac.at)
}

Received: 25 August 2012 - Published in Geosci. Instrum. Method. Data Syst. Discuss.: 3 September 2012

Revised: 13 March 2013 - Accepted: 22 March 2013 - Published: 12 April 2013

\begin{abstract}
A popular method for measuring the thermal conductivity of solid materials is the transient hot needle method. It allows the thermal conductivity of a solid or granular material to be evaluated simply by combining a temperature measurement with a well-defined electrical current flowing through a resistance wire enclosed in a long and thin needle. Standard laboratory sensors that are typically used in laboratory work consist of very thin steel needles with a large length-to-diameter ratio. This type of needle is convenient since it is mathematically easy to derive the thermal conductivity of a soft granular material from a simple temperature measurement. However, such a geometry often results in a mechanically weak sensor, which can bend or fail when inserted into a material that is harder than expected. For deploying such a sensor on a planetary surface, with often unknown soil properties, it is necessary to construct more rugged sensors. These requirements can lead to a design which differs substantially from the ideal geometry, and additional care must be taken in the calibration and data analysis.

In this paper we present the performance of a prototype thermal conductivity sensor designed for planetary missions. The thermal conductivity of a suite of solid and granular materials was measured both by a standard needle sensor and by several customized sensors with non-ideal geometry. We thus obtained a calibration curve for the non-ideal sensors. The theory describing the temperature response of a sensor with such unfavorable length-to-diameter ratio is complicated and highly nonlinear. However, our measurements reveal that over a wide range of thermal conductivities there is an almost linear relationship between the result obtained by the standard sensor and the result derived from the customized, non-ideal sensors. This allows for the measurement of thermal conductivity values for harder soils, which are not easily accessible when using standard needle sensors.
\end{abstract}

\section{Introduction}

Thermal conductivity is one of the key parameters required for modeling the thermal evolution of a planetary body and the interaction between the solid surface and subsurface layers and the atmospheric and radiative environment. While there exist methods to determine thermal parameters of a surface layer by remote measurements, e.g. by analyzing the irradiation emitted from the surface, these methods usually demand "ground truth" measurements that have to be performed inside the material, i.e. by an in situ method to allow for proper evaluation. The simplest way to do this is to insert a long and thin needle into the material to be measured and to heat this needle with a constant electrical power for a specified time. The thermal conductivity of the surrounding material can then be determined directly from the temperature increase of the needle as a function of time. According to the classical hot needle theory (see e.g. Healy et al., 1976), the temperature response of a needle inside a material which is heated by a constant power consists of two parts: an initial nonlinear phase which depends on conductivity and heat capacity and later on a phase where the temperature versus logarithm of time graph rises linearly and the inclination of the graph depends on heat conductivity only. Thus if the measurement time is long enough (from minutes to hours, depending on the material to be measured) the thermal conductivity can be evaluated without knowing the heat capacity of the material. The heat conductivity $k$ of the material can simply be determined by the formula

$k=\frac{Q}{4 \pi}\left(\frac{\mathrm{d} T}{\mathrm{~d} \ln t}\right)^{-1}$,

where $Q$ is the heating power supplied to the sensor in $\left[\mathrm{Wm}^{-1}\right]$ and $\left(\frac{\mathrm{d} T}{\mathrm{~d} \ln t}\right)$ is the measured temperature rise of the sensor as a function of the natural logarithm of time. 


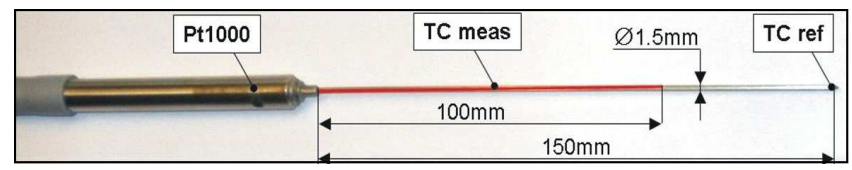

Fig. 1. The commercial TP02 (long needle) thermal conductivity probe produced by the Dutch company Hukseflux. The heated part is indicated in red.

However, such a simple evaluation is only possible for a very long and thin sensor needle with a length-to-diameter ratio of 60 or more, since the theory behind Eq. (1) assumes an infinitely thin and infinitely long line heat source. The reference sensor used here meets this requirement. ${ }^{1}$ However, sensors of this type are not rugged enough to be directly used in harder materials without pre-drilled holes (which may cause other errors for the evaluation of thermal conductivity) or on planetary surface missions, where the properties of the soil to be tested are generally unknown. A more rugged sensor is necessarily thicker and shorter and can no longer be considered as "long and thin". Therefore, Eq. (1) is no longer directly applicable.

In order to derive thermal conductivity values from measurements with this type of sensors, there are in principle two possibilities. Either a much more complicated formalism is used, applying the theory of "short and thick" sensors, or the same simple theory is used with an additional calibration function valid over the desired range of conductivities. The first method has been described in much detail in a recent paper by Hütter and Kömle (2012), in Hütter (2011) and most recently in Macher et al. (2013). The second method is the topic of the current paper. A more detailed description of the theoretical background can be found in Wechsler (1992) and in Kömle et al. (2011).

Very few thermal conductivity sensors have so far been successfully deployed in planetary missions. In the framework of the Apollo missions in the early 1970s, a few thermal conductivity measurements were performed on the lunar surface. However, evaluation was largely done along the first line, using the theory of multi-layered hollow cylindrical sensors (Langseth et al., 1972, 1973). Later on, in the frame of the Huyghens-Cassini mission, the thermal conductivity of Titan's atmosphere was determined by a probe working on the basis of the hot needle method (Hathi et al., 2007). However, the only space instrument that has measured thermal conductivity in the solid material of an extraterrestrial body other than the Moon was the TECP-instrument aboard the NASA Phoenix spacecraft, which landed on the Martian polar plains in 2008 (Zent et al., 2009, 2010). The method used to evaluate the thermal conductivity measurements obtained from the TECP-instrument is described in Cobos et

\footnotetext{
${ }^{1}$ Hukseflux Thermal Sensors - TP02 NON-STEADY STATE PROBE FOR THERMAL CONDUCTIVITY MEASUREMENTS: User Manual www.hukseflux.com
}

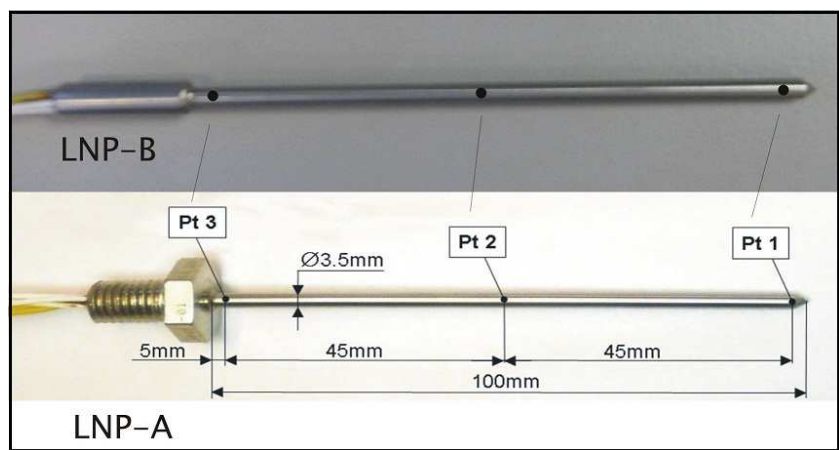

Fig. 2. The custom-made LNP-sensors fabricated by Hukseflux for use on planetary surfaces like on the Moon or Mars. They are heated over the whole length.

al. (2006). The needles used for the TECP measurements had an even greater deviation from the "ideal" geometry than the sensors described in this paper. It may be useful to compare their results with our findings in order to further validate the use of this calibration method for future heat conduction measurements on planetary surfaces. In the following sections we give a description of the sensors used for our calibration measurements, characterize the samples used and discuss the results obtained.

\section{Description of sensors}

\subsection{Reference sensor}

As reference sensor we have used an off-the-shelf thermal conductivity probe manufactured by the Dutch company Hukseflux (Type TP02). This sensor is shown in Fig. 1. According to the handbook, it is suitable for standard measurements in the range $0.1-6 \mathrm{Wm}^{-1} \mathrm{~K}^{-1}$ with an accuracy of $\pm 3 \%$ in the final thermal conductivity value. The needle has a diameter of $1.5 \mathrm{~mm}$ and a total length of $15 \mathrm{~cm}$. The uppermost $10 \mathrm{~cm}$ is actively heated during a measurement. The needle temperature in the heated part and in the unheated tip is measured by two thermocouples. With a lengthto-diameter ratio of 66 , this sensor fulfils the requirements for an "easy" evaluation of the thermal conductivity without the need for additional calibration.

\subsection{Prototype ruggedized sensors}

Two slightly different prototypes of custom-made sensors (LNP-A and LNP-B) were tested, and are shown in Fig. 2. They differ only in one detail: LNP-A has a mounting stud with a screw thread at the top which could be used to mount it onto a deployment device (for example a robotic arm on a planetary lander spacecraft). Because of the small dimensions of the needle such a part could influence the measurements due to its relatively large mass and heat capacity. 


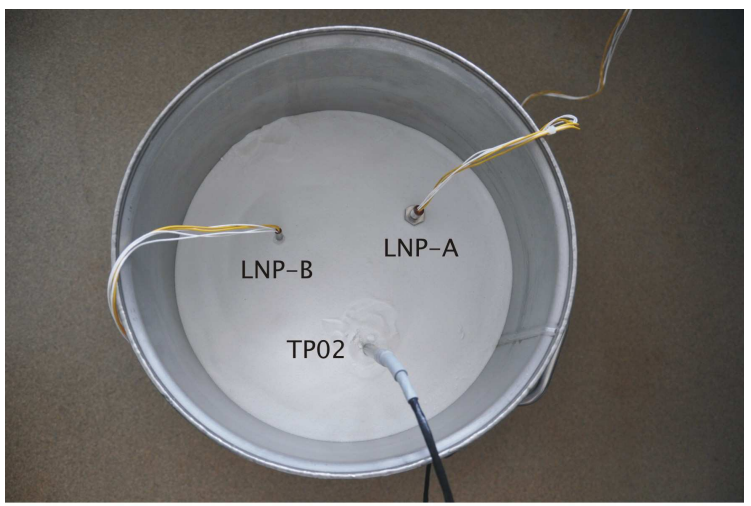

Glass beads sample

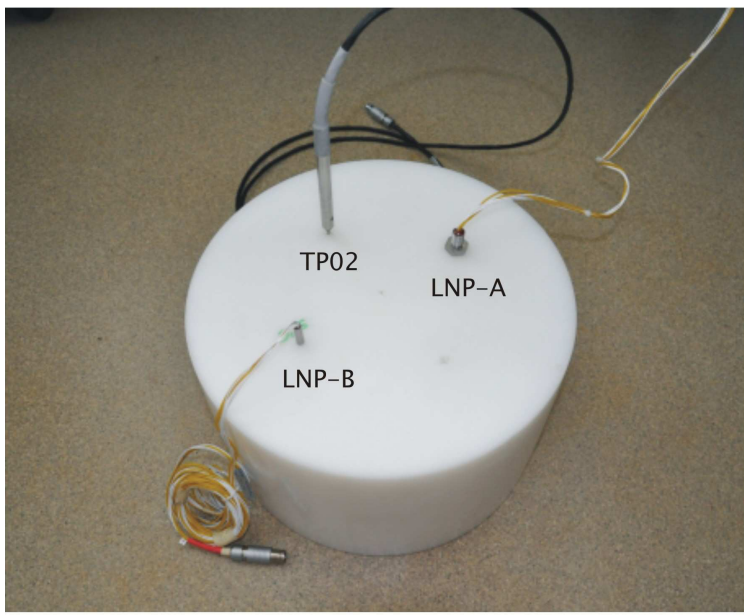

PE sample

Fig. 3. Experimental setup used for the calibration measurements. Top: sensors inserted into glass beads sample; bottom sensors inserted into PE sample.

Therefore, a second prototype (LNP-B) was built, which consisted only of the needle and the necessary connection wires without such a mounting stud. Both sensors were also built by Hukseflux and had a needle length of $100 \mathrm{~mm}$ and a diameter of $3.5 \mathrm{~mm}$. This implies a length-to-diameter ratio of 28. In these sensors the heating wire inside the needle extends over the whole needle length of $100 \mathrm{~mm}$. Temperature is recorded at three positions, as indicated in Fig. 2: in the center of the needle, close to the tip and close to the upper end. The readings of the central sensor are used for the evaluation of the thermal conductivity value. The temperature sensors are platinum resistance thermometers (PT1000) and temperatures are measured with a 4 -wire technique. ${ }^{2}$ Due to their larger diameter and their shorter length these sensors

\footnotetext{
${ }^{2}$ For an explanation of the four wire measurement technique with platinum resistance thermometers refer, for example, to the National Instruments webpage http://www.ni.com/white-paper/7115/ en.
}
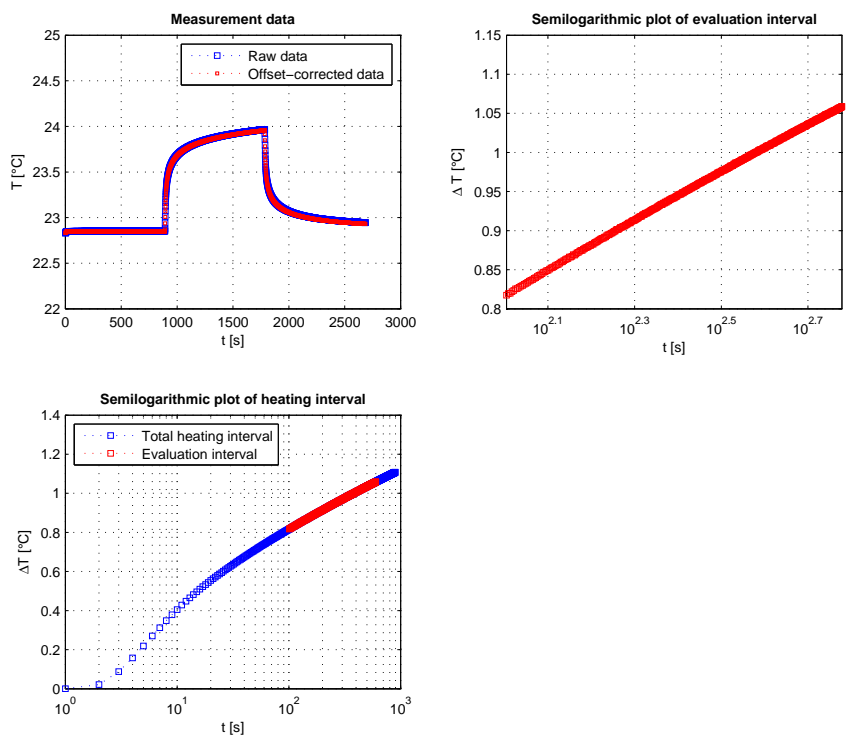

Fig. 4. Illustration of the thermal conductivity evaluation procedure using a Kerafol-KP96 measurement with sensor LNP-B as an example (heating time: $900 \mathrm{~s}$; heating power: $0.215 \mathrm{~W}$ ). Top left: raw data and offset-corrected data of the total measurement (including the decline phase of the temperature which is not used for the evaluation). Bottom left: semi-logarithmic plot of the heating interval. Top right: semi-logarithmic plot of the interval used for the thermal conductivity evaluation.

are by far more robust than the TP02 and easily withstand penetration into harder soils without damage.

\section{Characterization of calibration samples}

The calibration materials used for our measurements have been selected in order to cover the range of thermal conductivities from $10^{-1}$ to about $2 \mathrm{Wm}^{-1} \mathrm{~K}^{-1}$. The lower end corresponds to granular materials under normal pressure. As a representative of such a kind of material we have chosen silica glass beads with a grain size in the range $0.25-0.5 \mathrm{~mm}$. For the range $0.3-0.5 \mathrm{Wm}^{-1} \mathrm{~K}^{-1}$ the solid plastic material polyethylene (PE) was used. The range $0.5-0.6 \mathrm{Wm}^{-1} \mathrm{~K}^{-1}$ is typical for the thermal conductivity of water. However, since water is a fluid, it may undergo convection when heated by the sensor, which strongly increases the heat transfer between sensor and sample and therefore would lead to large errors in the determined thermal conductivity. To circumvent this problem, a small amount of agar (50 grams per liter of water) is added and dissolved in the water. This mixture is then heated up and boiled for several minutes. Upon cooling the solution back to room temperature a transparent, highly viscous gel is obtained, in which any form of convection is suppressed. However, its thermal properties are the same as those of water. When the agar is frozen and kept in a thermally stable environment, one obtains another useful 


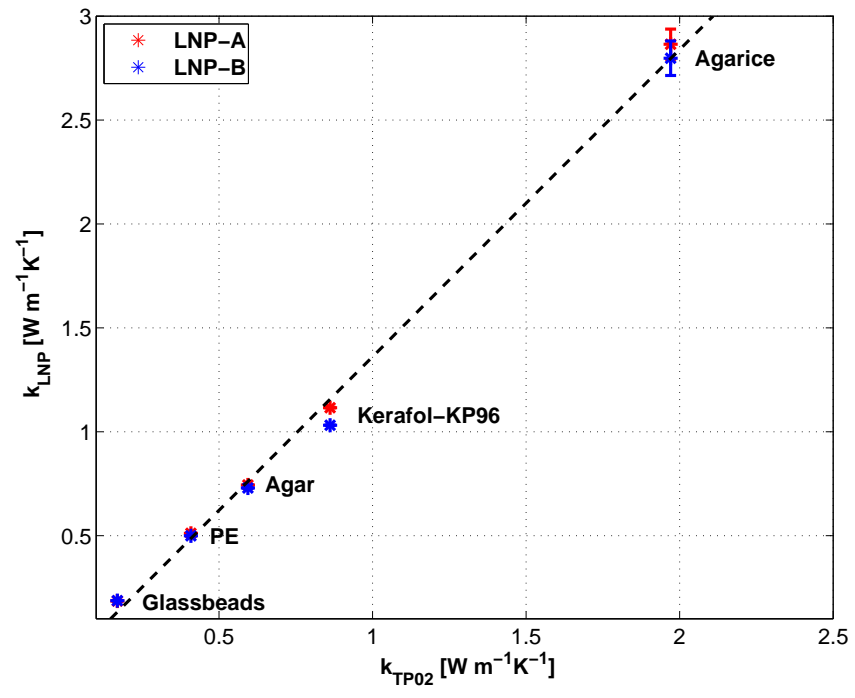

Fig. 5. Calibration of the custom-made LNP-sensors versus the commercial Hukseflux TP02 thermal conductivity sensor, which is considered as the reference sensor. The symbols represent the average measurement values obtained from the two LNP probes, while the dotted line is a linear fit between the measurement values using the data from both LNP-sensors.

calibration material, with $k \approx 2 \mathrm{Wm}^{-1} \mathrm{~K}^{-1}$, i.e. at the upper limit of our range of interest. To obtain the frozen agar sample, the agar samples measured before at room temperature were placed into a deep-freezer with the measurement needles inserted and kept there for several days, until a constant homogeneous temperature around $-20^{\circ} \mathrm{C}$ was reached. This led to a slight expansion of the sample analogous to the freezing of pure water, but the thermal contact of the needles to the surrounding ice remained well established, no major cracks were formed during the freezing process which would have compromised the thermal contact. This was possible because the containers used were somewhat flexible so that, in addition to the upward expansion, a volume expansion both towards the sides and towards the bottom was possible. Since the temperature changes due to sensor heating never exceeded a few degrees, there was no danger that local phase changes of the material along the sensor/sample could have occurred.

There is a lack of easily accessible materials in the range between 0.7 and $2 \mathrm{Wm}^{-1} \mathrm{~K}^{-1}$. To fill this gap, we used a bulk of thermally conductive grease as sample material (KerafolKP96). This grease is viscous enough that convection is suppressed, but at the same time soft enough that sensors can be easily inserted. In our calibration measurements we have used a thermal grease with a bulk thermal conductivity around $1 \mathrm{Wm}^{-1} \mathrm{~K}^{-1}$ to bridge the gap between PE and water (agar) ice.

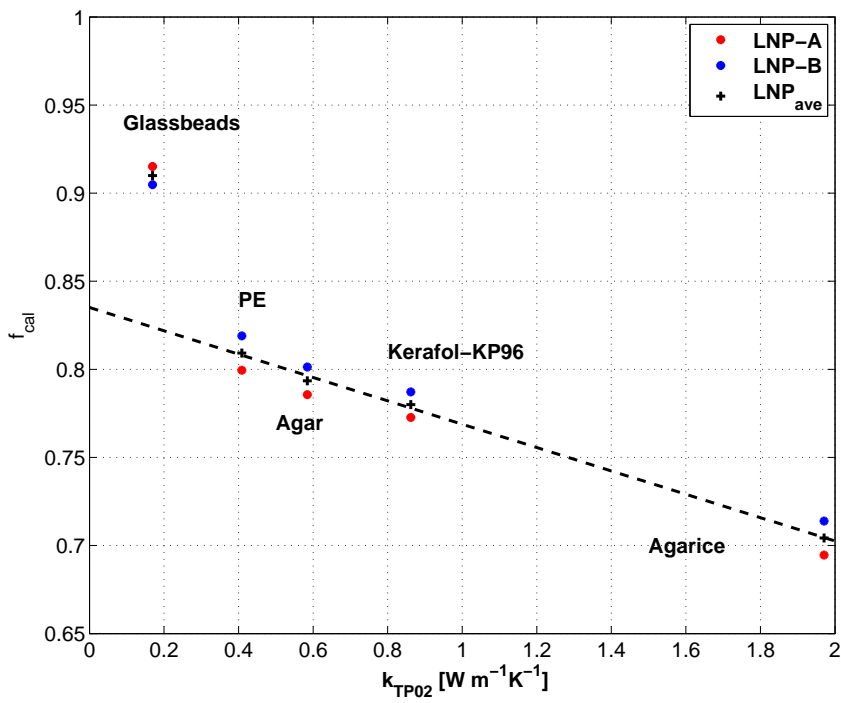

Fig. 6. Plot of the calibration factors for the different materials as listed in Table 2. The dashed line is a linear fit through the measurement values excluding the glass beads. $\mathrm{LNP}_{\mathrm{ave}}$ is the average of the measured LNP-A and LNP-B values for each calibration material.

\section{Calibration of the ruggedized sensors}

For the calibration of the custom-made sensors the following procedures were performed:

- Reasonably large-sized samples were prepared, which were big enough in diameter and height that all three sensors could be inserted without disturbing each other during a measurement and making sure that no influence from the sample boundaries could disturb the measurements. For estimating minimum sample sizes, refer to the formulae given in Hütter and Kömle (2012).

- The samples (with sensors inserted) were kept for at least several hours in a thermally stable environment to make sure that they were isothermal at the beginning of a measurement series. All measurements were performed at room temperature, i.e. at an ambient temperature in the range $20-25^{\circ} \mathrm{C}$, apart from those in agarice, where the samples were stored in a deep-freezer at $-22^{\circ} \mathrm{C}$.

- Thermal conductivity measurements were made by heating each sensor separately, allowing for long enough time periods between two subsequent measurements (at least several hours). The chosen heating periods of the sensors were between 300 and 900 s, depending on the sample used.

- The thermal conductivity was evaluated using the following standard procedure, as illustrated in Fig. 4:

1. Removal of any temperature trend from the data not associated with the active heating of the sensor. 
Table 1. Thermal conductivity measurement results for the different calibration materials and sensors.

\begin{tabular}{|c|c|c|c|c|c|}
\hline Sensor $/ \lambda$ & $\begin{array}{r}\text { Glassbeads } \\
\mathrm{Wm}^{-1} \mathrm{~K}^{-1}\end{array}$ & $\begin{array}{c}\mathrm{PE} \\
\mathrm{Wm}^{-1} \mathrm{~K}^{-1}\end{array}$ & $\begin{array}{c}\text { Agar } \\
\mathrm{Wm}^{-1} \mathrm{~K}^{-1}\end{array}$ & $\begin{array}{l}\text { Kerafol KP96 } \\
\mathrm{Wm}^{-1} \mathrm{~K}^{-1}\end{array}$ & $\begin{array}{c}\text { Agarice } \\
\mathrm{Wm}^{-1} \mathrm{~K}^{-1}\end{array}$ \\
\hline \multirow[t]{9}{*}{ ТP02 } & 0.1684 & 0.4126 & 0.5874 & 0.8477 & 2.1064 \\
\hline & 0.1707 & 0.4479 & 0.6251 & 0.8709 & 1.9281 \\
\hline & 0.1688 & 0.3755 & 0.5938 & 0.8743 & 1.9631 \\
\hline & $\ldots . .$. & 0.4560 & 0.6145 & 0.8554 & 1.8860 \\
\hline & $\ldots \ldots$ & 0.3680 & 0.6033 & $\ldots . .$. & $\ldots . .$. \\
\hline & $\ldots \ldots$ & 0.3779 & 0.6165 & $\ldots \ldots$. & $\ldots \ldots$ \\
\hline & $\ldots \ldots$ & 0.4190 & $\ldots . .$. & $\ldots \ldots$. & $\ldots \ldots$ \\
\hline & $\ldots \ldots$ & 0.4127 & $\ldots \ldots$ & $\ldots \ldots$. & $\ldots \ldots$ \\
\hline & 0.1693 & 0.4087 & 0.6068 & 0.8621 & 1.9709 \\
\hline \multirow[t]{5}{*}{ LNP-A } & 0.1841 & 0.5125 & 0.7396 & 1.1125 & 2.7702 \\
\hline & 0.1859 & 0.5119 & 0.7529 & 1.1201 & 2.8757 \\
\hline & 0.1850 & 0.5092 & 0.7462 & 1.1161 & 2.7835 \\
\hline & ...... & ...... & 0.7373 & 1.1139 & 2.9208 \\
\hline & 0.1850 & 0.5112 & 0.7440 & 1.1157 & 2.8375 \\
\hline \multirow[t]{6}{*}{ LNP-B } & 0.1865 & 0.4979 & 0.7328 & 1.0957 & 2.7807 \\
\hline & 0.1868 & 0.4998 & 0.7228 & 1.1000 & 2.7039 \\
\hline & 0.1879 & 0.4994 & 0.7336 & 1.0918 & 2.6502 \\
\hline & $\ldots .$. & $\ldots .$. & 0.7255 & 1.0931 & 2.8554 \\
\hline & $\ldots \ldots$ & $\ldots \ldots$ & $\ldots . .$. & $\ldots .$. & 2.8146 \\
\hline & 0.1871 & 0.4990 & 0.7274 & 1.0951 & 2.7609 \\
\hline
\end{tabular}

Table 2. Calibration factors derived for the different materials and sensors.

\begin{tabular}{cccccc}
\hline Sensor/Material & Glassbeads & PE & Agar & Kerafol-KP96 & Agarice \\
\hline LNP-A & 0.9151 & 0.7995 & 0.7856 & 0.7727 & 0.6946 \\
LNP-B & 0.9048 & 0.8190 & 0.8013 & 0.7872 & 0.7139 \\
\hline
\end{tabular}

2. Identification of the suitable interval of the measured temperature profile (linear part on the $T$ versus $\ln t$ graph).

3. Calculation of the thermal conductivity according to Eq. (1).

The general setup of the measurements is shown in Fig. 3 for two of the used samples, the glass beads and the PEblock. The agar sample was prepared in the same $30 \mathrm{~cm}$ diameter steel container as the glass beads sample. For performing the measurements in the agar-ice, this sample was placed in a deep freezer with the sensors inserted and frozen at a temperature of $-22^{\circ} \mathrm{C}$. In this way it was ensured that the sensor needles were firmly frozen into the ice and thus had a good contact to the sample with negligible thermal resistance. For the Kerafol-KP96 sample the sample container was smaller (diameter of $16 \mathrm{~cm}$ ) but still large enough to ensure that there was no influence of the container walls on the measurement results. The active heating times of the sensors used for the thermal conductivity measurements were typically $900 \mathrm{~s}$. The heating powers were in the range
$100-500 \mathrm{~mW}$, depending on the estimated conductivity of the sample material.

\section{Results}

The results of our calibration measurements with the ruggedized thermal conductivity sensors are summarized in Table 1 and in Fig. 5. All measurements were repeated two times or more. The average value from the individual results was used to calculate the calibration factor (italic numbers in the table). The scattering of the measurement results can be seen from Table 1 and from the error bars in Fig. 5. Note that the 2-sigma error bars are based on only a few data samples (3-6) per measurement point. Thus we do not claim that they are the result of a significant statistical analysis, which would have demanded an unrealistically large number of measurements for each material. Rather we show them here to illustrate the good repeatability of the performed measurements and the fact that both of the two ruggedized sensors (despite of having a slightly different geometry) give consistent 
results. Actually, the error bars are only visible on the graph for the agar-ice sample, where the quality is poorer than for the other measurements, which were done at room temperature in a thermally stable room (The reason for this is not clear, but it may have to do with the temperature regulation cycles of the the cooling device, which overlay the heating of the sensors during a measurement or with other unknown disturbances).

Using these measured average values from both types of sensors (the reference sensor TP02 and the ruggedized sensors LNP-A and LNP-B) a correction factor was calculated per sample and hence conductivity range. This allows the true thermal conductivity to be obtained from a measurement with one of the ruggedized sensors according to the formula:

$k_{\mathrm{TP} 02}=f_{\mathrm{cal}} \cdot k_{\mathrm{LNP}}$.

The calibration factors derived for the different materials and sensors (LNP-A and LNP-B) as calculated from the measurements are listed in Table 2. As can be seen from Fig. 5, in general the measured thermal conductivity values over the range of interest can be well fitted by a linear relationship (constant calibration factor, dotted line). The average value of the individual calibration factors as given in Table 2 is $f_{\text {cal }}=0.799 \approx 0.8$. This would lead to a maximum error of $15 \%$ over the thermal conductivity range covered by the measurements. However, this is not a statistical error only, as can be seen by a closer look on Table 2. Plotting the individual $f_{\mathrm{cal}}$-values versus the measured $k_{\mathrm{TP} 02}$-values (Fig. 6) indicates a linear decreasing trend in the range 0.4$2 \mathrm{Wm}^{-1} \mathrm{~K}^{-1}$, but the derived values for the glass beads tend to be closer to the reference value than the linear trend in the rest of the curve would suggest. Further tests exploring the trend at lower thermal conductivities are therefore necessary and planned in a next step.

\section{Conclusions}

The measurements reveal that the prototype sensors give consistently higher values of the thermal conductivity when evaluated in the same way as the measurements with the standard sensor. However, we found that the data produced by the ruggedized sensors could be well fitted by assuming a linear relation between the values obtained by the standard sensors and the ruggedized sensors. This result confirms that measurements with the rugged prototype sensors, which have strongly non-ideal geometry, can be made for any unknown material (in the appropriate thermal conductivity range) by applying a constant calibration factor.
Acknowledgements. This paper is a late result of the project L317-N14 supported by the Austrian Fonds zur Förderung der wissenschaftlichen Forschung. The custom-made sensors used for the measurements described here were designed and manufactured under the funding of this project.

Edited by: P. Falkner

\section{References}

Cobos, D. R., Campbell, G. S., and Campbell, C. S.: Modified line heat source for measurement of thermal properties on Mars, in: Proceedings of the 28th International Thermal Conductivity Conference, edited by: Dinwiddie, R. D., White, M. A., and McElroy, D. L., 331-338, Destech. Publ., Lancaster, Pa, 2006.

Hathi, B., Daniell, P. M., Banaszkiewicz, M., Hagermann, A., Leese, M. R., and Zarnecki, J. C.: Thermal conductivity instrument for measuring planetary atmospheric properties and data analysis technique, J. Therm. Anal. Calorim. 87, 585-590, 2007.

Hütter, E. S.: Development and testing of thermal sensors for planetary applications, PhD Thesis, Karl-Franzens- Universität Graz, 2011.

Hütter, E. S. and Kömle, N. I.: Performance of thermal conductivity probes for planetary applications, Geosci. Instrum. Method. Data Syst., 1, 53-75, doi:10.5194/gi-1-53-2012, 2012.

Kömle, N. I., Hütter, E. S., Macher, W., Kaufmann, E., Kargl, G., Knollenberg, J., Grott, M., Spohn, T., Wawrzaszek, R., Banaszkiewicz, M., and Hagermann, A.: In situ methods for measuring thermal properties and heat flux on planetary bodies, Planet. Space Sci., 59, 639-660, 2011.

Langseth, M. G. J., Clark, S. P. J., Chute, J. L. J., Kheim, S. J. J., and Wechsler, A. E.: Heat flow experiment, in: Apollo 15: Preliminary science report, (NASA SP-289), 1972.

Langseth, M. G. J., Kheim, S. J. J., and Chute, J. L. J.: Heat flow experiment, in: Apollo 17: Preliminary science report, (NASA SP-330), 1973.

Macher, W., Kömle, N. I., Bentley, M. S., and Kargl, G.: The heated infinite cylinder with sheath and two thermal surface resistance layers, Int. J. Heat Mass Transf., 57, 528-534, 2013.

Wechsler, A. E.: The probe method for measurement of thermal conductivity, in: Compendium of Thermophysical Property Measurement Methods, edited by: Maglić, H. D., Cezairliyan, A., and Peletsky, V. E., 161-185, Plenum Press, New York, 1992.

Zent, A. P., Hecht, M. H., Cobos, D. R., Campbell, G. S., Campbell, C. S., Cardell, G., Foote, M. C., Wood, S. E., and Mehta, M.: Thermal and Electrical Conductivity Probe (TECP) for Phoenix, J. Geophys. Res., 114, E00A27, doi:10.1029/2007JE003052, 2009.

Zent, A. P., Hecht, M. H., Cobos, D. R., Wood, S. E., Hudson, T. L., Milkovich, S. M., DeFlores, L. P., and Mellon, M. T.: Initial results from the thermal and electrical conductivity probe (TECP) on Phoenix, J. Geophys. Res., 115, E00E14, doi:10.1029/2009JE003420, 2010. 